\title{
Effects of Electrical Stimulation of Olivocochlear Fibers in Cochlear Potentials in the Chinchilla
}

\author{
Diego Elgueda ${ }^{1}$, Paul H. Delano ${ }^{1,2}$, and Luis Robles ${ }^{1}$ \\ ${ }^{1}$ Programa de Fisiología y Biofisica, Instituto de Ciencias Biomédicas (ICBM), Facultad de Medicina, Universidad de Chile, \\ Independencia 1027 Casilla 70005, Correo 7 Santiago, Chile \\ ${ }^{2}$ Servicio Otorrinolaringología, Hospital Clínico Universidad de Chile, Santiago, Chile
}

Received: 14 December 2010; Accepted: 2 February 2011; Online publication: 2 March 2011

\begin{abstract}
The mammalian cochlea has two types of sensory cells; inner hair cells, which receive auditory-nerve afferent innervation, and outer hair cells, innervated by efferent axons of the medial olivocochlear (MOC) system. The role of the MOC system in hearing is still controversial. Recently, by recording cochlear potentials in behaving chinchillas, we suggested that one of the possible functions of the efferent system is to reduce cochlear sensitivity during attention to other sensory modalities (Delano et al. in J Neurosci 27:4146-4153, 2007). However, in spite of these compelling results, the physiological effects of electrical MOC activation on cochlear potentials have not been described in detail in chinchillas. The main objective of the present work was to describe these efferent effects in the chinchilla, comparing them with those in other species and in behavioral experiments. We activated the MOC efferent axons in chinchillas with sectioned middle-ear muscles by applying current pulses at the fourth-ventricle floor. Auditory-nerve compound action potentials (CAP) and cochlear microphonics $(\mathrm{CM})$ were acquired in response to clicks and tones of several frequencies, using a round-window electrode. Electrical efferent stimulation produced CAP amplitude suppressions reaching up to $11 \mathrm{~dB}$. They were higher for low to moderate sound levels. Additionally, CM amplitude increments were found, the largest $(\leq 2.5 \mathrm{~dB})$ for low intensity tones. CAP suppression was present at all
\end{abstract}

Correspondence to: Luis Robles - Programa de Fisiología y Biofísica, Instituto de Ciencias Biomédicas (ICBM), Facultad de Medicina • Universidad de Chile $\cdot$ Independencia 1027 Casilla 70005, Correo 7 Santiago, Chile. Telephone: +56 (2) 978-6042; fax: +56 (2) 777-6916; email: 1robles@med.uchile.cl stimulus frequencies, but was greatest for $2 \mathrm{kHz}$. CM increments were highest for low-frequency tones, and almost absent at high frequencies. We conclude that the effect obtained in chinchilla is similar to but smaller than that observed in cats, and that the effects seen in awake chinchillas, albeit different in magnitude, are consistent with the activation of efferent fibers.

Keywords: auditory efferent, olivocochlear, chinchilla, electric stimulation, cochlear potentials

\section{INTRODUCTION}

In mammals, the auditory efferent system originates in the auditory cortex, projects to the inferior colliculus and the superior olivary complex, and reaches the cochlea through the olivocochlear (OC) fibers (for review, see Robles and Delano 2008). These fibers are identified according to their place of origin as lateral or medial OC fibers (Warr and Guinan 1979). The lateral OC subsystem originates in the lateral superior olivary nucleus, and projects unmyelinated axons that innervate afferent dendrites in the region beneath inner hair cells. The medial olivocochlear (MOC) fibers emerge from the ventral nucleus of the trapezoid body, and make synaptic contact at the basolateral region of outer hair cells $(\mathrm{OHC})$.

Electrical activation of MOC fibers at their midline crossing towards the contralateral cochlea in the fourth-ventricle floor reduces cochlear sensitivity (Galambos 1956; Fex 1959; Desmedt 1962; Gifford and Guinan 1987). As MOC fibers directly innervate OHCs, their activity modifies the input impedance 
and membrane potential of OHCs (Housley and Ashmore 1991), affecting the mechanical feedback that they provide to basilar membrane motion (Mountain 1980; Siegel and Kim 1982; Murugasu and Russell 1996; Cooper and Guinan 2003). These changes reduce the cochlear-amplifier gain, producing a decrease in auditory-nerve compound action potentials (CAP) and an increase in cochlear microphonics (CM) amplitudes that reflects a higher voltage drop caused by the increase of current flow through OHCs and the cochlear circuit (Guinan 1996).

Among various proposed roles for the auditory efferent system, based on experiments performed in behaving animals, it has been suggested that the olivocochlear system modulates cochlear sensitivity during attentional processes. Cochlear potentials recorded in cats and chinchillas performing a visual discrimination task, showed amplitude reductions of auditory-nerve CAPs during periods of visual attention. In behaving cats, CAP reductions occurred with no significant changes in CM amplitude (Oatman 1971), while in behaving chinchillas CAP reductions were accompanied by $\mathrm{CM}$ amplitude increases (Delano et al. 2007).

The aforementioned changes in cochlear sensitivity observed in behaving animals are presumably produced by activation of auditory efferent fibers, as electrical MOC activation in anesthetized cats produces analogous, but larger, CAP reductions and CM augmentations (Gifford and Guinan 1987). Discrepancies between the effects observed in behaving cats and chinchillas could be explained by specific differences in the anatomy of the efferent system between mammals, or by the influence of awake versus anesthetized condition on MOC activity.

MOC-activation effects on cochlear responses have been mainly studied in cat and guinea pig (Galambos 1956; Desmedt 1962; Gifford and Guinan 1987; Murugasu and Russell 1996); however, evidence showing significant differences in the anatomy and distribution of OC neurons between the chinchilla and other mammals (Iurato et al. 1978; Azeredo et al. 1999) makes it important to measure these efferent effects in this widely used hearing model. The aim of this work is to report the effects of electrical stimulation of MOC fibers on cochlear potentials (i.e., $\mathrm{CAP}$ and $\mathrm{CM}$ ) in the anesthetized chinchilla, comparing them with those observed in other commonly used laboratory animals (e.g., cats, guinea pigs).

\section{METHODS}

\section{Animals and anesthesia}

All procedures involving animals were made in accordance with the institutional Bioethics Committee and
NIH Guidelines for the Care and Use of Laboratory Animals, publication No. 86-23, revised 1996. In this study, 14 adult chinchillas (Chinchilla laniger) weighing 400-700 g were used. They were anesthetized with xylazine (4-8 mg/kg, I.M.), ketamine (20-40 mg/kg, I.M.) and atropine (0.04 mg/kg, I.M.) and were maintained deeply anesthetized by repeating half doses every 30-45 minutes, or when necessary, judged by the foot-withdrawal reflex. Rectal temperature was maintained at $35-37^{\circ} \mathrm{C}$ by means of a heating pad. At the end of every experiment, deeply anesthetized animals were humanely euthanized with an overdose of sodium thiopental $(120 \mathrm{mg} / \mathrm{kg})$.

\section{Surgical procedures}

The left pinna was resected for proper access to the external auditory meatus and tympanic membrane, and a dorsal opening was made in the bulla to allow cutting of the tensor tympani muscle. The cochlea was accessed by a posterior aperture of the tympanic bulla, and a silver-wire electrode was placed at the round window. This approach allowed us to detach the stapedius muscle from its insertion in eight of the 14 animals used. The fourth ventricle was exposed by dorsal craniotomy and aspiration of the anterior portion of the cerebellar vermis. Hemorrhage and accumulation of cerebrospinal fluid was controlled with haemostatic sponge.

\section{Stimulus and data acquisition}

All experiments were performed in a double-walled sound-attenuating room, and on top of a floating table, isolated from external noises and vibrations. Animals were placed on a stereotaxic apparatus that allowed micrometric movements of the stimulating electrodes. Acoustic stimuli were clicks (produced by $100-\mu$ s-wide pulses) with a peak intensity of $100 \mathrm{~dB}$ SPL and 15 ms duration tones of 1, 2, 4, 6, 8, 10 and $12 \mathrm{kHz}$. All stimuli were generated by a RP2.1 real-time processor $(100,000$ points/s), attenuated by a PA-5 programmable attenuator and delivered with an EC1 electrostatic speaker (Tucker-Davis Technologies, System 3) through a closed ear cavity sealed to the external auditory meatus. A Knowles microphone was inserted into the ear cavity to perform sound pressure level calibrations.

Cochlear electrical potentials were acquired through an $80 \mu \mathrm{m}$ diameter silver wire electrode placed on the round window, amplified $80 \mathrm{~dB}$ and filtered (300-10,000 and 300-20,000 Hz for low- and high-frequency tones respectively). These signals were digitized at 40,000 samples/s with a data acquisition board (National Instruments, 6024E) housed in a desktop PC. 
The efferent pathway was stimulated at the midline crossing in the floor of the fourth ventricle with an array of six Nichrome ${ }^{\circledR}$ electrodes $(200 \mu \mathrm{m}$ diameter) separated by $500 \mu \mathrm{m}$, which allowed bipolar stimulation. Each electrode was coated with enamel, leaving uncovered $500 \mu \mathrm{m}$ at the tip. Stimulation electrodes were positioned with a hydraulic micro-drive (David Kopf Instruments 1207B), and kept isolated from the recording system with a custom-made optic coupler. The pair of electrodes that produced the largest reduction of CAP amplitude while causing just noticeable facial motor responses was used (Gifford and Guinan 1987; Guinan and Stankovic 1996). Facial motor responses are due to the proximity of the efferent fiber decussation to the genua of the facial nerve.

Efferent stimulation parameters used were: $300-\mu \mathrm{s}-$ wide pulses presented at frequencies of 200-300 pulses/s in periods of $100 \mathrm{~ms}$, since similar parameter values have yielded the greatest cochlear sensitivity reductions in other species (Gifford and Guinan 1987; Cooper and Guinan 2003). Variation of these parameters in our initial experiments produced little effect on our results.

Each trial consisted of three consecutive series: control, efferent and recovery. Two paradigms were used: A, series consisting of 64 presentations of clicks or tones at a $1 \mathrm{~Hz}$ rate, and $\mathrm{B}$, series of 128 presentations at a $3 \mathrm{~Hz}$ rate. In the efferent series acoustic stimuli, were preceded (30 ms) by pulse bursts delivered through the pair of electrodes at the fourth ventricle (Fig. 1).

\section{Data analysis}

Acoustical stimuli were presented with alternated polarity, in order to allow us to separate CAPs from CMs. Responses to stimuli of each polarity were independently averaged, and later added together to cancel CMs and isolate CAP responses. CAP amplitudes were calculated between the peaks of the $\mathrm{N}_{1}$ and $\mathrm{P}_{1}$ waves in the averaged responses. The CAP latency was measured as the period between the presentation of the acoustic stimulus and the peak of the $\mathrm{N}_{1}$ wave. Subtraction of the averaged responses to stimuli of the two polarities allowed the computation of the amplitudes of CMs by performing a fast Fourier transform (FFT) in a $12.8 \mathrm{~ms}$ window that excluded the CAP response (custom-made $\mathrm{C}$ program, LabWindows/CVI environment). Efferent effects were measured by the reduction (or increase) in CAP (or CM) amplitude produced by the MOC electrical stimulation. CM amplitudes of single trials and the mean of two consecutive CAP trials were used to calculate the average and standard deviation of control and efferent periods. The significance of the differences between cochlear responses in control and efferent conditions were determined by paired $t$-tests.
A
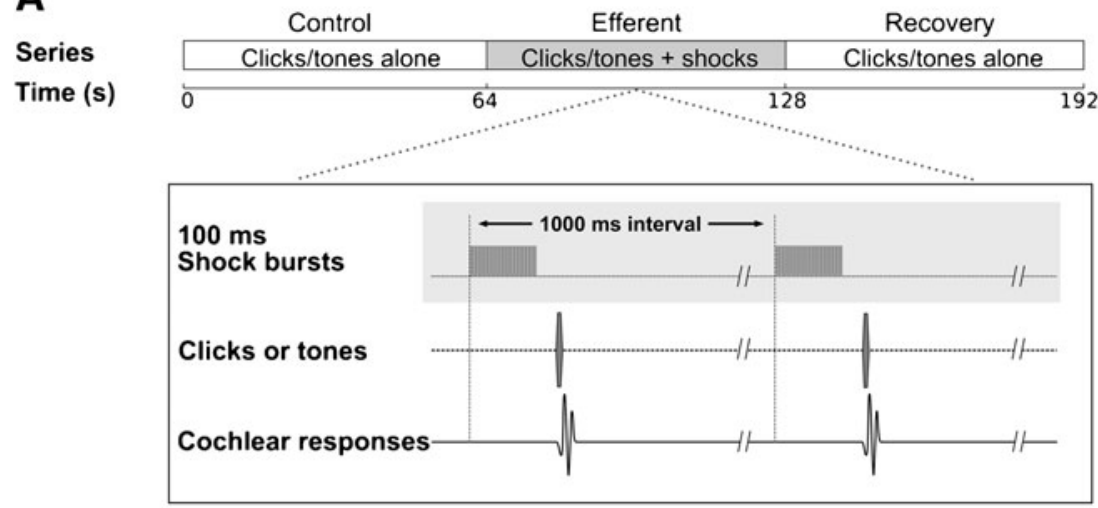

B

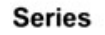

Time (s)
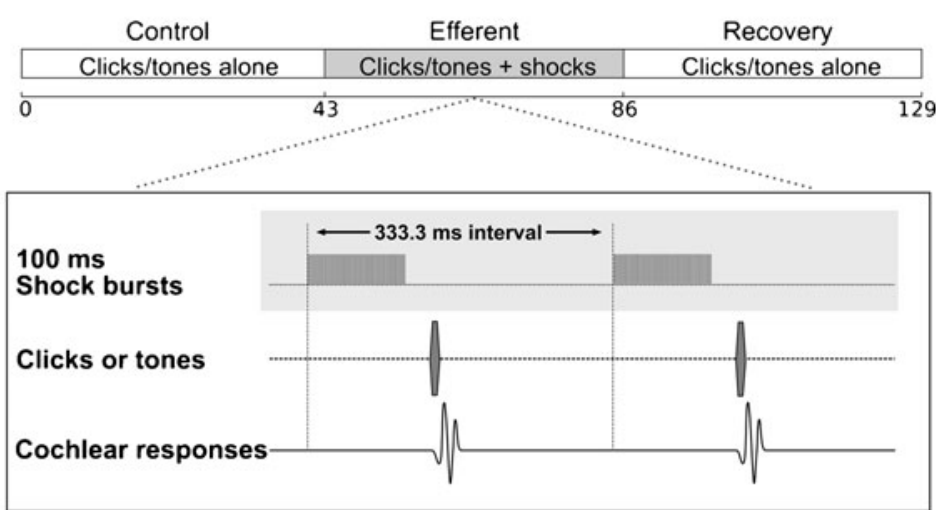

FIG. 1. Experimental paradigms. Each trial consisted of three consecutive series: control, efferent and recovery. In the efferent series, acoustic stimuli were preceded (30 ms) by shock bursts (100 ms) delivered in the fourth-ventricle floor. In the control and recovery series, stimuli were presented without efferent stimulation. Paradigm $\mathbf{A}$ had 64 stimulus presentations at a rate of $1 \mathrm{~Hz}$. Paradigm $\mathbf{B}$ had 128 stimulus presentations at a rate of $3 \mathrm{~Hz}$. Simultaneously, cochlear responses were recorded through an electrode placed on the round window. 
The temporal course of the efferent-effect strength along trials in the presence of electrical stimulation was evaluated by calculating the slope of a regression line for CAP and $\mathrm{CM}$ amplitudes $(\mu \mathrm{V} / \mathrm{s})$. One-way ANOVA was used to compare CAP and CM efferenteffect slopes obtained with stimuli of different frequencies. Tukey post-hoc test was used to evaluate significant differences between different frequencies. Pearson correlation indexes were calculated to evaluate sound pressure dependence of CAP and CM efferenteffect slopes.

\section{RESULTS}

The electrical pulses delivered at the floor of the fourth ventricle produced CAP amplitude reductions in all 14 chinchillas studied. In the experiments in which middle-ear muscles function was preserved $(n=6)$, we measured CAP reductions up to $26 \mathrm{~dB}$, and a maximum $\mathrm{CM}$ increase of $1.5 \mathrm{~dB}$, while in experiments in which middle-ear muscles were detached $(n=8)$, we measured CAP reductions up to $11 \mathrm{~dB}$ and $\mathrm{CM}$ increases up to $2.5 \mathrm{~dB}$. Results presented in the following sections were all obtained in the eight chinchillas in which possible middle-ear effects were eliminated by section of the tendon of the tensor tympani muscle and detachment of the stapedius muscle from its insertion.

\section{Efferent effects in cochlear responses}

In the eight chinchillas with detached middle-ear muscles, electrical stimulation of MOC efferent fibers produced reductions in CAP amplitude that were accompanied by CM enhancements (Fig. 2). These CAP reductions and $\mathrm{CM}$ enhancements were observed in responses to tones at various frequencies (1 to $12 \mathrm{kHz}$ ) and sound levels, and also in responses to clicks at several intensities. CAP amplitude reductions in response to tones and clicks were greater for acoustic stimuli at low and moderate intensities, reaching suppressions up to $11 \mathrm{~dB}$; while at higher sound levels CAP reductions were lower or absent (Figs. 3 and 4). Figure 4 displays CAP amplitude reductions as a function of stimulus intensities obtained in the different animals, confirming the consistency of the observed efferent effects. Concomitantly with CAP decreases, electrical activation of efferent fibers caused augmentations of CM amplitudes (Figs. 2 and 5). These efferent effects on CMs, however, were of lower magnitude than those seen in CAPs, reaching increases of up to $2.5 \mathrm{~dB}$. The increments in CM amplitude were strongly influenced by stimulus intensity; being highest at the lowest

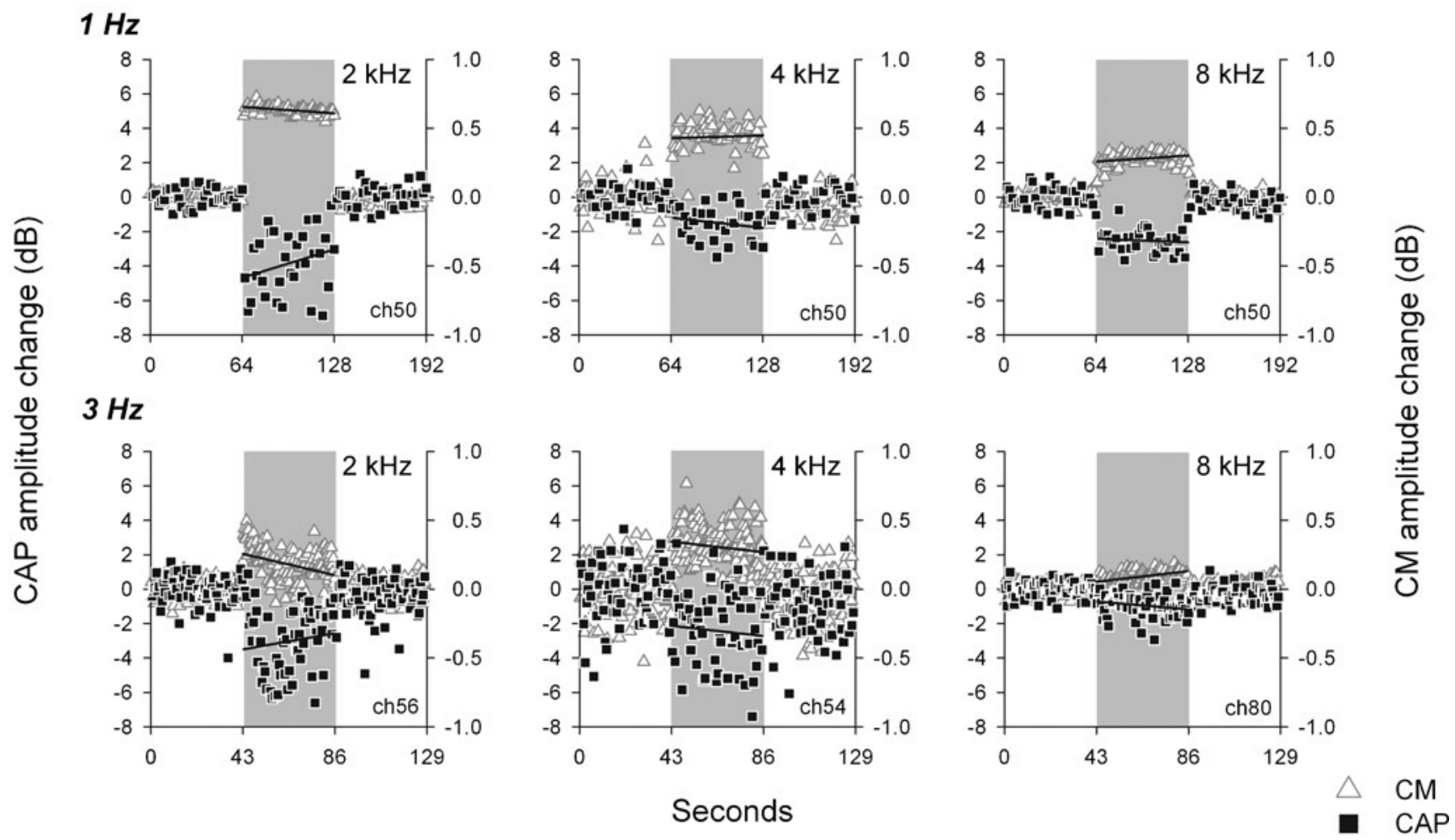

FIG. 2. Amplitude modulation of cochlear potentials by MOC efferent activation. Changes in amplitude of sound-evoked CAP (squares) and CM (triangles) produced by efferent electrical stimulation, expressed as dB with reference to controls. Efferent series are marked by the shaded area. Upper and lower rows correspond to experimental data obtained at $1 \mathrm{~Hz}$ and $3 \mathrm{~Hz}$ rates respectively. Lines in panels represent calculated regression lines of MOC effect vs time for each condition. 

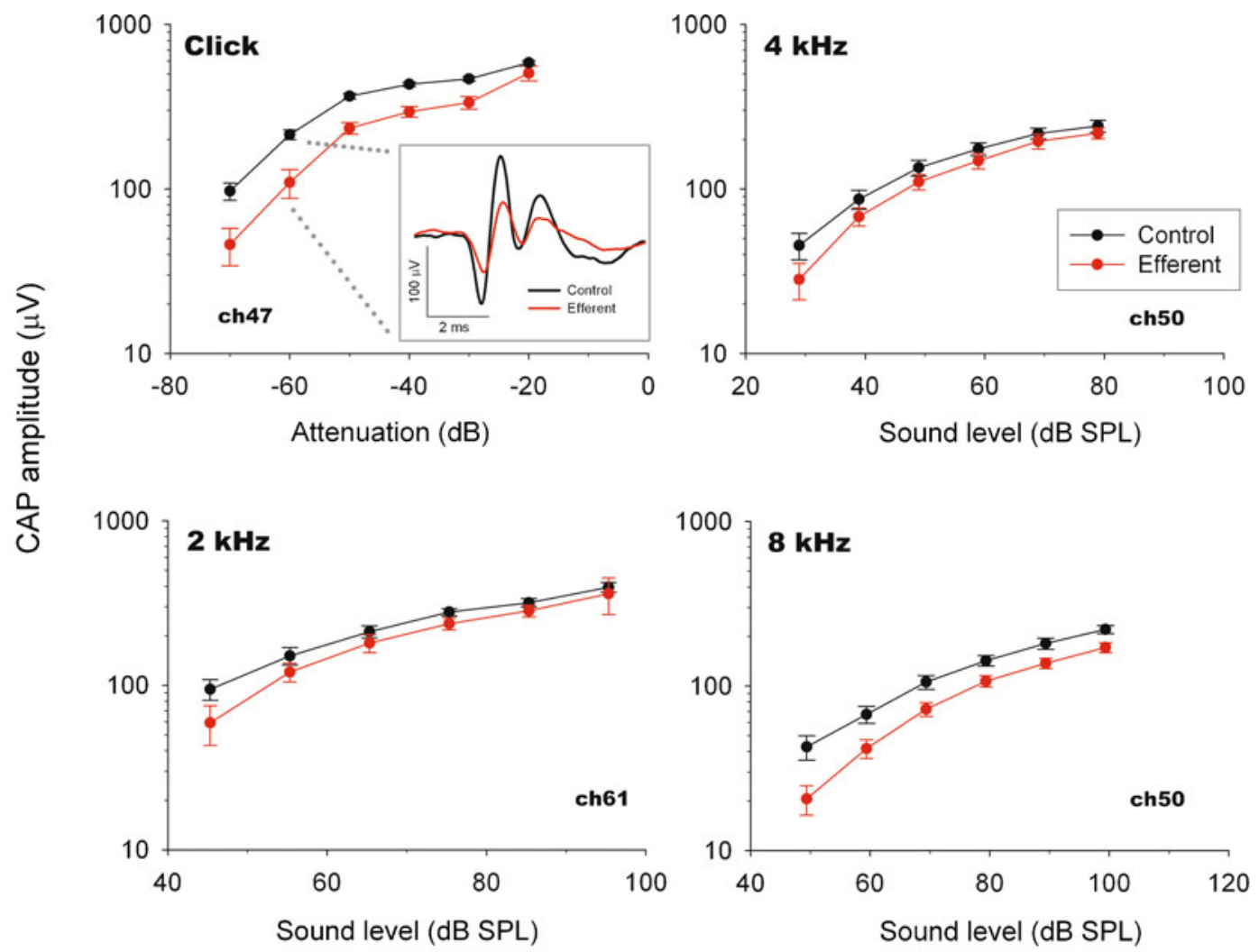

FIG. 3. CAP input-output curves. CAP amplitudes with (red) and without (black) efferent electrical stimulation as a function of acoustic stimulus intensity recorded in three chinchillas. Efferent-produced CAP reductions are greater at lower acoustic stimulus intensities. CAP traces with and without efferent activation are shown in inset box.
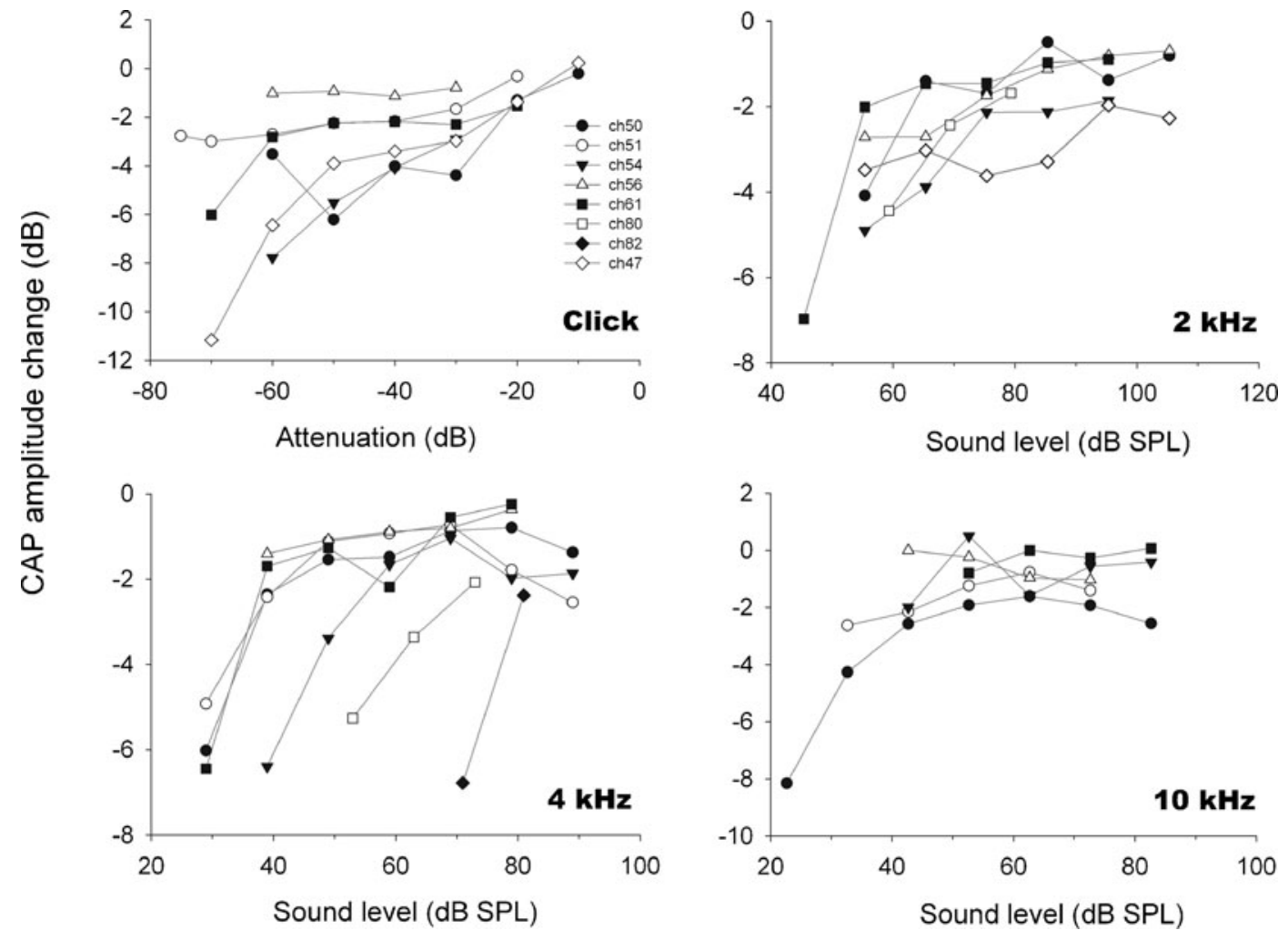

FIG. 4. CAP amplitude modulation as function of acoustic-stimuli intensity recorded in eight animals. Efferent effect magnitude is expressed as $\mathrm{dB}$ of change with reference to control. The efferent effect is larger for low intensity acoustic stimuli. 
$1 \mathrm{kHz}$
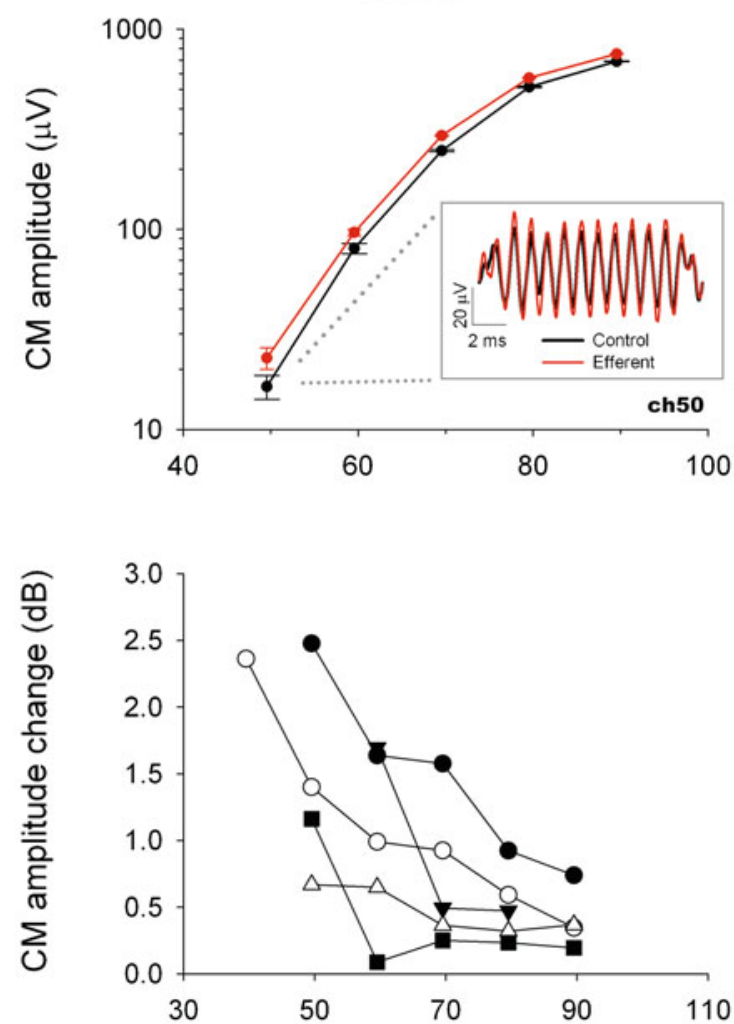

2 kHz

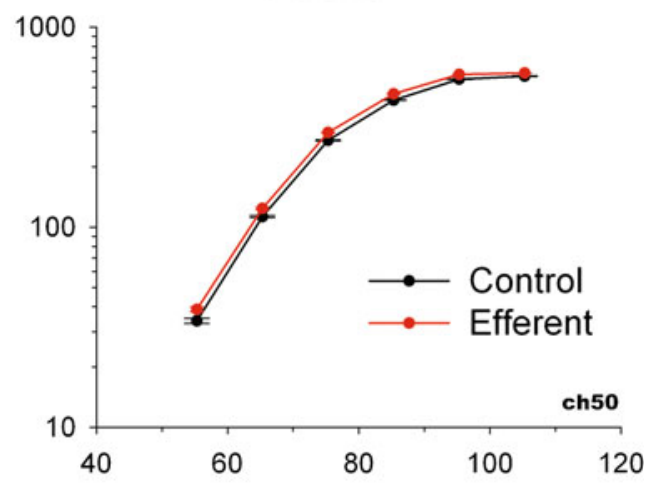

Sound level (dB SPL)

FIG. 5. Efferent effects on CM amplitudes. Upper panels: CM input-output curves obtained in one animal at two stimulus frequencies with (red) and without (black) efferent electrical stimulation. Inset shows an example of CM traces with and without efferent activation. Values were obtained through FFT analysis. Each symbol represents the mean \pm standard deviation of 128 single trials. All CM increments shown in this figure were statistically significant (paired $t$-test; $p<0.05$ ). Bottom panels: efferent $C M$ enhancements obtained in five chinchillas. CM increments were larger for low-frequency and low-intensity stimuli ( 1 and $2 \mathrm{kHz}$ and 40 to $60 \mathrm{~dB} \mathrm{SPL}$ ). Effects are expressed as dB of change with reference to control amplitude.

stimulus intensity levels and almost disappearing at higher levels (Fig. 5).

Both efferent effects, CAP suppression and CM enhancement, were also dependent on tone frequency. CAP suppression, although present at all tested stimulus frequencies, reached a maximum for $2 \mathrm{kHz}$ tones and had a secondary peak at frequencies around $8 \mathrm{kHz}$ (Figs. 3 and 6). CM enhancements reached a maximum increase for 1 to $2 \mathrm{kHz}$ tones, and showed no amplitude changes or even suppressions at higher frequencies (Fig. 6).

Efferent stimulation also produced increases in CAP latency. These were present at all tested frequencies, and were strongly dependent on stimulus intensity (Fig. 7), reaching response delays of up to $0.2 \mathrm{~ms}$ at the lowest sound pressure levels.

\section{Temporal course of the efferent effect}

To assess the temporal variation in strength of the efferent effect, we calculated the slope of a regression line for CAP and CM amplitudes along trials in the presence of efferent activation, as exemplified in Figure 2. A positive CM efferent-effect slope represents an increase in strength of the efferent effect with time, while a positive CAP efferent-effect slope represents a decrease in strength of the efferent effect.

As shown in Figure 2, there was high variability in the values of CAP efferent-effect slopes, ranging from negative to positive values $(-1.91$ to $1.18 \mu \mathrm{V} / \mathrm{s})$, while most values of CM efferent-effect slopes were negative or close to zero ( -0.91 to $0.10 \mu \mathrm{V} / \mathrm{s})$. Thus, in the majority of the experiments, $\mathrm{CM}$ increases displayed a reduction in the strength of the effect along efferent trials. There was a significant difference between the CAP efferenteffect slopes obtained with the 1 and $3 \mathrm{~Hz}$ rate protocols $[0.022 \pm 0.160$ and $0.155 \pm 0.427 \mu \mathrm{V} / \mathrm{s}$ respectively (mean \pm S.D.); unpaired $t$-test, $p<0.01]$. A significant difference was also found between the CM efferent-effect slopes observed with the 1 and $3 \mathrm{~Hz}$ rate protocols $(-0.003 \pm$ 0.022 and $-0.082 \pm 0.151 \mu \mathrm{V} / \mathrm{s}$ respectively; unpaired $t$-test, $p<0.01)$. 
A

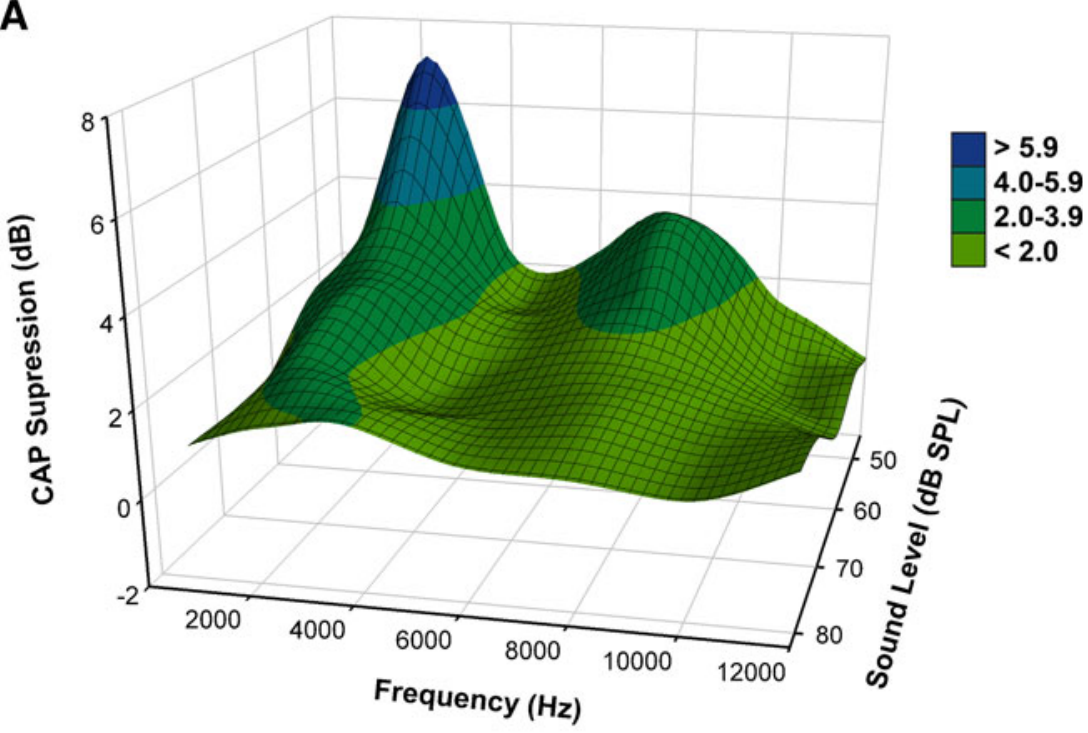

FIG. 6. Influence of stimulus intensity and frequency in MOC effects in cochlearpotentials amplitudes obtained in eight experiments. A Grand average of CAP suppression effects as a function of frequency and sound pressure level. A main peak effect is observed for low frequencies around $2 \mathrm{kHz}$ and a smaller peak for frequencies around $8 \mathrm{kHz}$. B Grand average of $\mathrm{CM}$ amplitude augmentation effects (obtained through FFT) as a function of frequency and sound pressure level. Larger $\mathrm{CM}$ augmentations are observed for low frequencies ( 1 and $2 \mathrm{kHz}$ ) at low sound pressures, while no changes or even small $(<1.0 \mathrm{~dB})$, but significant reductions are seen for frequencies above $4 \mathrm{kHz}$.

\section{B}

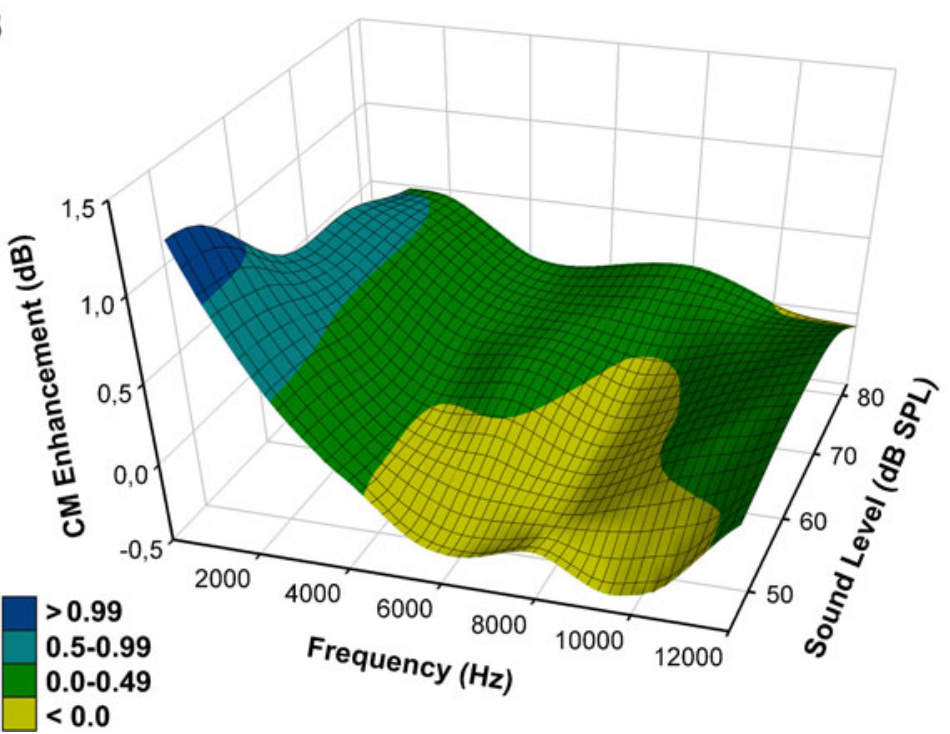

Figure 8 displays the mean values of CAP and CM efferent-effect slopes obtained for the different stimulus frequencies using the 1 and $3 \mathrm{~Hz}$ rate protocols. The figure shows that, only for the $3 \mathrm{~Hz}$ protocol, the mean slopes of the CAP and CM efferent effect depended on stimulus frequency, as the absolute mean values of efferent-effect slopes decreased from low- to highfrequency stimuli (CAP ANOVA, F=5.84, $p<0.01$; CM ANOVA, $\mathrm{F}=4.14, p<0.01$; see legend of Figure 8 for post-hoc tests). There was no significant correlation between the magnitudes of CAP and CM efferent-effect slopes obtained at different sound pressure levels (Pearson test).

\section{DISCUSSION}

Electrical activation of efferent fibers in the chinchilla (with detached middle-ear muscles) produced CAP decreases of up to $11 \mathrm{~dB}$, concomitantly with $\mathrm{CM}$ increases of up to $2.5 \mathrm{~dB}$. In the experiments in which we did not detach middle-ear muscles, electrical stimulation produced CAP suppressions that reached up to $26 \mathrm{~dB}$, which suggested that, in those experiments, the electric stimulation produced not only olivocochlear activation, but also facial-nerve activation that reduced the cochlear input by stapedius-muscle contractions. To avoid middle-ear effects, in this report we only discuss results obtained in animals with sectioned middle-ear muscles.

\section{Efferent modulation of cochlear potentials}

There was considerable inter-animal variability in the magnitudes of the observed efferent effects that, as suggested for other species, most likely reflects variability in the activity level of efferent MOC neurons (Liberman 1989). However, this variability 

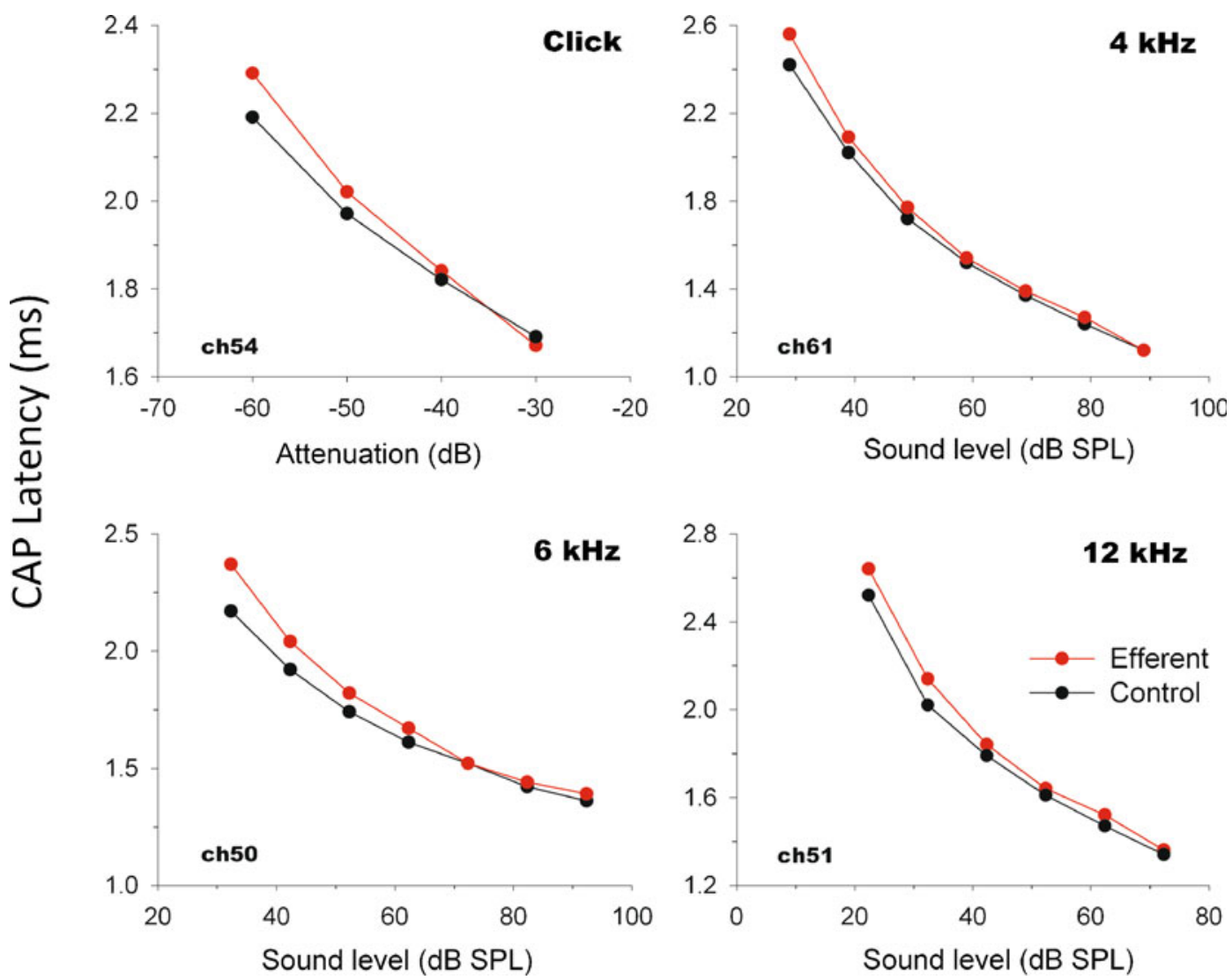

FIG. 7. CAP latency modulation with (red) and without (black) MOC activation as a function of acoustic stimulus intensity. The epoch between the presentation of the acoustic stimulus and the peak of the CAP first wave was increased by activation of MOC fibers.

could partially originate in differences in the positioning of the stimulating electrodes in the brain stem.

As mentioned in Methods, in this work we have measured MOC effects by the reduction (or increase) in CAP (or CM) amplitude produced by the efferent stimulation. However, in other reports the metric for MOC effects has been the amount by which sound

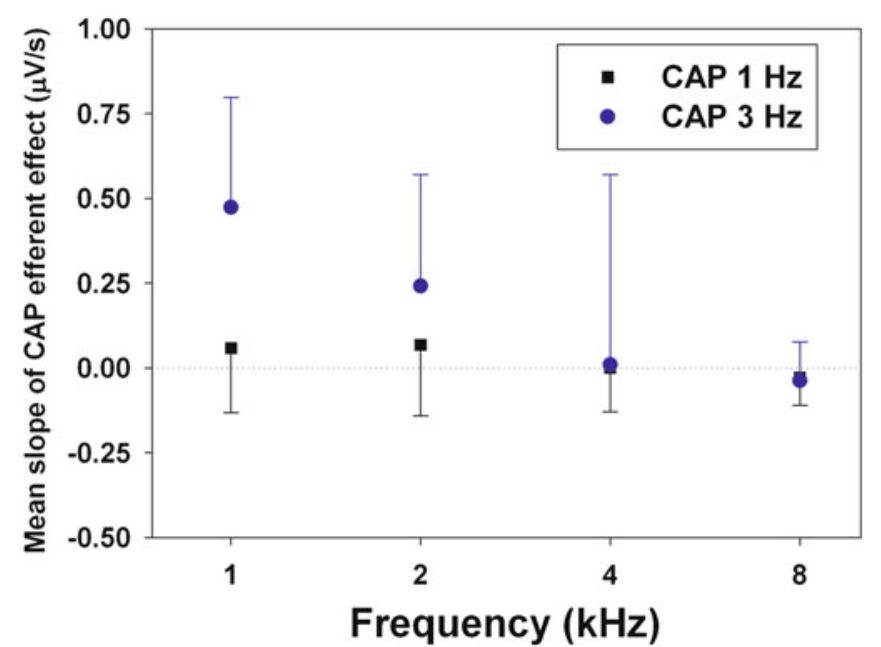

level has to be increased during efferent stimulation to produce a response of the same magnitude as that without MOC stimulation. All comparisons of our results with previous data are made using our metric. The efferent-induced CAP reductions and CM enhancements that we found in the chinchilla displayed similar characteristics to those already

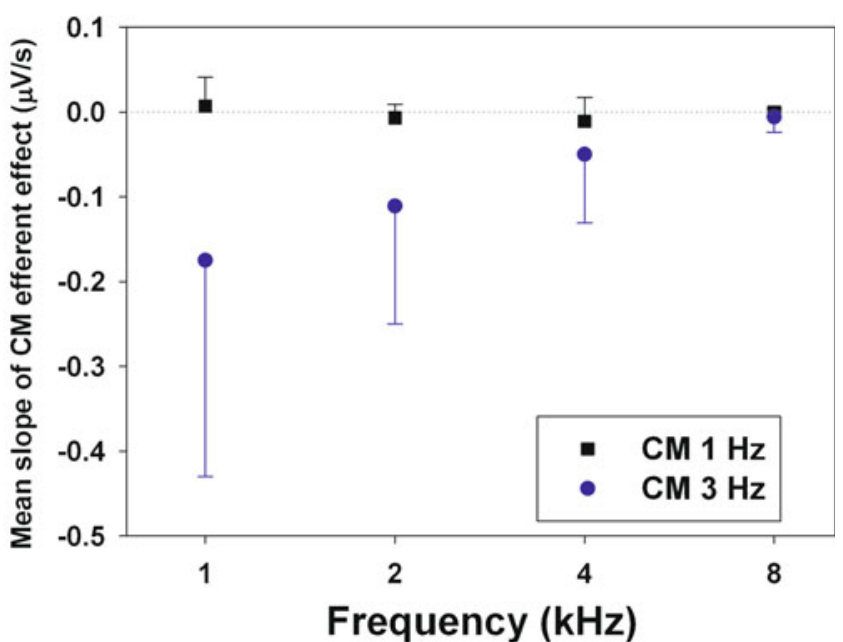

FIG. 8. Mean slopes of regression lines of MOC effect vs time on CAP and CM responses. Mean slopes in $\mu \mathrm{V} / \mathrm{s}$ (and standard deviations) were calculated for the different stimulus frequencies presented with the 1 and $3 \mathrm{~Hz}$ rate protocols. Significant differences between CAP and CM efferent-effect slopes were found only for the $3 \mathrm{~Hz}$ protocol. A Tukey post-hoc test showed significant differences between 1 and $8 \mathrm{kHz}$ stimuli for CAP and CM efferent-effect slopes, and for 1 and $4 \mathrm{kHz}$ stimuli for CAP efferent effects. 
reported for the cat (Galambos 1956; Fex 1959; Desmedt 1962; Sohmer 1965; Wiederhold and Peake 1966; Gifford and Guinan 1987) and the guinea pig (Sridhar et al. 1995; Murugasu and Russell 1996), although in most cases they were of smaller magnitudes. In our measurements, we found efferent-produced CAP reductions of up to a maximum of $11 \mathrm{~dB}$, compared with reductions near or over $20 \mathrm{~dB}$ observed in cats (Desmedt and Monaco 1961; Wiederhold 1970; Gifford and Guinan 1987). These differences between the effects observed by us in chinchilla and by other authors in other species suggest that chinchillas have a functionally weaker MOC system.

Efferent-induced CAP amplitude reductions were accompanied by CM increases, although the former were consistently larger than the latter (see Figs. 2 and $6)$; this result is in agreement with previous reports in other species (Desmedt and Monaco 1961; Sohmer 1965; Gifford and Guinan 1987; Murugasu and Russell 1996). We obtained maximum CAP reductions for $2 \mathrm{kHz}$, a frequency corresponding to a location at about the middle of the chinchilla cochlea (Eldredge et al. 1981). This peak in the strength of MOC effect for frequencies represented at about the center of the cochlea is also found in other species. In cat, guinea pig and mouse, most MOC effects on both CAPs and auditory-nerve fibers, and also the anatomical distribution of MOC fibers, display a single broad peak in the mid-frequencies; at $4-10 \mathrm{kHz}$ in cat (Liberman et al. 1990; Guinan and Gifford 1988a), at $7-10 \mathrm{kHz}$ in guinea pig (Teas et al. 1972), and at about $10 \mathrm{kHz}$ in mouse (Maison et al. 2003). However, our finding of another smaller peak of CAP efferent inhibition for frequencies around $8 \mathrm{kHz}$ was unexpected (discussed in next section). In chinchilla, the only anatomical study available on efferent fiber distribution shows that most MOC fibers innervate the two more basal cochlear turns (Iurato et al. 1978), a distribution that is compatible with the MOC effects we observed at the different frequencies.

Even though there was correlation between the magnitudes of MOC effects observed in CAP and CM in the different animals (as shown in Fig. 9), the two effects reached their maxima for different stimulus frequencies. In fact, this is not unexpected, since it is known that round-window $\mathrm{CM}$ potentials represent a weighted vectorial sum of $\mathrm{CM}$ contributions that weighs more heavily the more basal generators (Dallos 1973; Cheatham et al. 2011), while CAPs elicited by low-intensity tones reflect activity generated by a limited number of cochlear fibers, with characteristic frequencies close to the stimulus frequency (Özdamar and Dallos 1976).

The observed efferent-induced increases in CAP latency are similar to those described in cat (Gifford and Guinan 1987) and are consistent with the

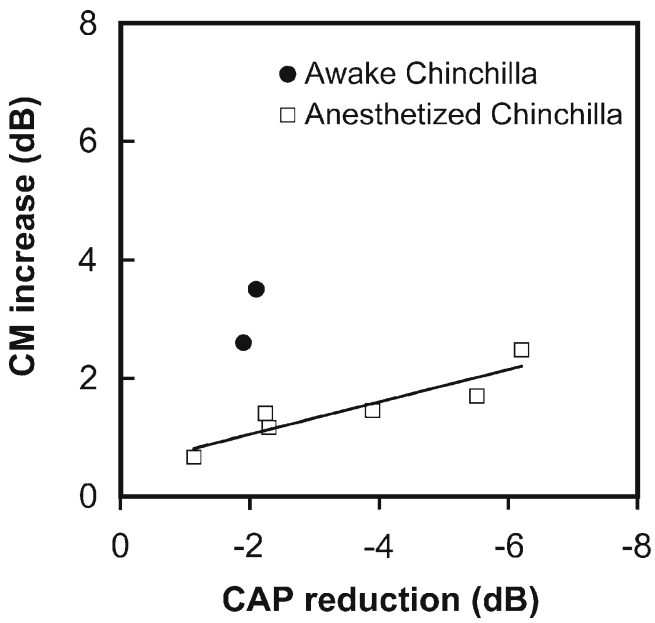

FIG. 9. Comparison of click-evoked CAP reductions vs CM increases in behaving and anesthetized chinchillas. Each point indicates average of the maximum values of CAP reduction and CM increase obtained for each animal at 50-70 dB SPL. CM increases were obtained for 2 and $1 \mathrm{kHz}$ tones in awake and anesthetized chinchillas respectively. Data for awake animals were extracted from Table 2 in Delano et al. 2007. Note larger CAP reductions for anesthetized chinchillas, in contrast with larger $C M$ increments for behaving chinchillas. There is correlation between the magnitudes of CAP suppression and CM augmentation in anesthetized chinchillas $(p<0.05$, Pearson test. Equation for regression line: $\left.\mathrm{Y}=-0.2741 * \mathrm{X}+0.5037 ; R^{2}=0.8377\right)$.

decreases in tuning sharpness produced in single auditory nerve fibers by MOC activation (Guinan and Gifford 1988b).

\section{Temporal course of the efferent effect}

Two types of MOC efferent effects have been described; fast effects that take place with time constants in the order of tens of milliseconds, and slow effects that occur with time constants three orders of magnitude larger (Sridhar et al. 1995; Cooper and Guinan 2003). Our experimental paradigm was best suited to measure fast MOC effects by evaluating the change in amplitude of cochlear potentials recorded at the onset of the efferent stimulation series $(30 \mathrm{~ms}$ after delivery of electric pulses at the floor of the fourth ventricle). However, the presence of slow MOC effects was recognized by the increase of the efferent effect during the stimulation period (CAP reductions displaying negative slopes, mostly for frequencies $\geq 4 \mathrm{kHz}$ ).

As mentioned in Results (Fig. 8), there was a significant decrease in absolute magnitude of CAP and CM efferent-effect slopes for increasing stimulus frequencies presented with the $3 \mathrm{~Hz}$-rate protocol. For stimulus frequencies of 1 and $2 \mathrm{kHz}$, CAP efferenteffect slopes had positive values, indicating a decrease of MOC effect with time, while for $8 \mathrm{kHz}$ stimuli CAP efferent-effect slopes were negative, indicating an 
increase of MOC effect with time. These negative slopes of the efferent-induced CAP reductions would indicate the presence of slow MOC effects for highfrequency stimuli. A similar dependence of fast and slow effects on stimulus frequency has been observed in guinea pig (Sridhar et al. 1995), in which the fast effect had a larger peak around $8-10 \mathrm{kHz}$, while the slow effect had a smaller peak for higher frequencies (around $14 \mathrm{kHz}$ ). As mentioned above, we found the greatest CAP decrease around $2 \mathrm{kHz}$, and a secondary peak of CAP reduction near $8 \mathrm{kHz}$ that could reflect both, fast and slow effects in the chinchilla.

\section{Comparison with efferent modulations}

\section{in behaving animals}

In a previous study, we found significant CAP reductions and $\mathrm{CM}$ increases in behaving chinchillas during periods of selective attention to visual stimuli (Delano et al. 2007). We suggested that those changes in cochlear sensitivity were produced by activation of MOC efferent fibers. In the present study we found similar changes by stimulating MOC efferent fibers in anesthetized chinchillas. Efferent-mediated CAP suppressions produced in both conditions were accompanied by simultaneous CM enhancements, which reached maxima at similar stimulus frequencies $(1-2 \mathrm{kHz})$. These results suggest that the modulations in cochlear sensitivity previously observed in chinchillas performing a visuospatial task are consistent with an activation of MOC efferent fibers during the periods of selective visual attention.

Figure 9 compares the maximum values of CAP reduction and $\mathrm{CM}$ increase obtained for different animals in the two conditions: awake performing the visual task, and anesthetized under efferent stimulation. As shown in the figure, maximum CAP reductions, measured at similar sound pressure levels, were larger in the present experiments $(6 \mathrm{~dB})$ than those previously observed during periods of visual attention in behaving chinchillas $(2 \mathrm{~dB})$. This would seem to contradict the evidence showing that efferent suppression is weaker in anesthetized than in awake guinea pigs (Guitton et al. 2004), and that evoked otoacoustic emissions increase in ketamine anesthetized chinchillas, which also indicates a ketamine-induced reduction of MOC activity (Harel et al. 1997). However, the stronger CAP reductions produced, in this study, in some of the animals by efferent electrical stimulation is probably explained by a higher number of efferent fibers recruited, as well as by higher firing rates elicited on these fibers in this condition than during selective visual attention.

Unexpectedly, CM increments were significantly lower in the present experiments than in the awake condition (up to $2.5 \mathrm{~dB}$ versus $3.5 \mathrm{~dB}$, see Fig. 9). It is possible that the increase in efferent effect on the CAP, in contrast with the decrease of effect on the $\mathrm{CM}$, observed in the present work as compared to the efferent effects reported in behaving chinchillas could be explained by the completely different conditions of activation of the efferent system in the awake animal. Finally, the weaker efferent effects on CM in our anesthetized animals are also supported by a model of chronically de-efferented awake chinchillas, in which CM amplitudes decreased by $6 \mathrm{~dB}$ after sectioning the OC fibers (Zheng et al. 2000).

In conclusion, the present results: (1) demonstrate that electrical activation of MOC fibers in the anesthetized chinchilla produces CAP reductions and CM increases similar, but smaller than those observed in cats, (2) suggest that the MOC activity that regulates $\mathrm{CM}$ and $\mathrm{CAP}$ responses is modulated differentially in the awake/anesthetized states of the subjects, and (3) imply that the modulations in cochlear sensitivity produced in behaving chinchillas by selective visual attention (Delano et al. 2007) are produced by activation of MOC efferent fibers.

\section{ACKNOWLEDGMENTS}

We thank Fernando Vergara for technical assistance and FONDECYT 1080227 and Fundación Guillermo Puelma for financing.

\section{REFERENCES}

Azeredo WJ, Kliment ML, Morley BJ, Relkin E, Slepecky NB, Sterns A, Warr WB, Weekly JM, Woods CI (1999) Olivocochlear neurons in the chinchilla: a retrograde fluorescent labelling study. Hear Res 134:57-70

Cheatham MA, Naik K, Dallos P (2011) Using the cochlear microphonic as a tool to evaluate cochlear function in mouse models of hearing. J Assoc Res Otolaryngol 12(1):113-125

Cooper NP, Guinan JJ JR (2003) Separate mechanical processes underlie fast and slow effects of medial olivocochlear efferent activity. J Physiol 548:307-312

Dallos P (1973) The auditory periphery, chapter 5. Academic, New York

Delano PH, Elgueda D, Hamame CM, Robles L (2007) Selective attention to visual stimuli reduces cochlear sensitivity in chinchillas. J Neurosci 27:4146-4153

Desmedt JE (1962) Auditory-evoked potentials from cochlea to cortex as influenced by activation of the efferent olivo-cochlear bundle. J Acoust Soc Am 34:1478-1496

Desmedt JE, Monaco P (1961) Mode of action of the efferent olivocochlear bundle on the inner ear. Nature 192:1263-1265

Eldredge DH, Miller JD, Bohne BA (1981) A frequency-position map for the chinchilla cochlea. J Acoust Soc Am 69:10911095

FEx J (1959) Augmentation of cochlear microphonic by stimulation of efferent fibres to the cochlea; preliminary report. Acta Otolaryngol 50:540-541 
Galambos R (1956) Suppression of auditory nerve activity by stimulation of efferent fibers to cochlea. J Neurophysiol 19:424-437

GifFord ML, Guinan JJ JR (1987) Effects of electrical stimulation of medial olivocochlear neurons on ipsilateral and contralateral cochlear responses. Hear Res 29:179-194

Guinan JJ JR (1996) Physiology of Olivocochlear Efferents. In: Dallos P, Popper A, Fay R (eds) The cochlea. Springer, New York, pp $435-502$

Guinan JJ JR, GIFFORD ML (1988a) Effects of electrical stimulation of efferent olivocochlear neurons on cat auditory-nerve fibers. I. Rate-level functions. Hear Res 33:97-114

Guinan JJ JR, GIFFord ML (1988b) Effects of electrical stimulation of efferent olivocochlear neurons on cat auditory-nerve fibers. III. Tuning curves and thresholds at CF. Hear Res 37:29-46

Guinan JJ JR, Stankovic KM (1996) Medial efferent inhibition produces the largest equivalent attenuations at moderate to high sound levels in cat auditory-nerve fibers. J Acoust Soc Am 100:1680-1690

Guitton MJ, Avan P, Puel JL, Bonfils P (2004) Medial olivocochlear efferent activity in awake guinea pigs. NeuroReport 15:1379-1382

Harel N, Kakigi A, Hirakawa H, Mount RJ, Harrison RV (1997) The effects of anesthesia on otoacoustic emissions. Hear Res 110:25-33

Housley GD, Ashmore JF (1991) Direct measurement of the action of acetylcholine on isolated outer hair cells of the guinea pig cochlea. Proc Biol Sci 244:161-167

Iurato S, Smith CA, Eldredge DH, Henderson D, Carr C, Ueno Y, Cameron S, Richter R (1978) Distribution of the crossed olivocochlear bundle in the chinchilla's cochlea. J Comp Neurol 182:57-76

Liberman MC (1989) Rapid assessment of sound-evoked olivocochlear feedback: suppression of compound action potentials by contralateral sound. Hear Res 38:47-56

Liberman MC, Dodds LW, Pierce S (1990) Afferent and efferent innervation of the cat cochlea: quantitative analysis with light and electron microscopy. J Comp Neurol 301:443-4460

Maison SF, Adams JC, Liberman MC (2003) Olivocochlear innervation in the mouse: immunocytochemical maps, crossed versus uncrossed contributions, and transmitter colocalization. J Comp Neurol 455:406-416
MountaIn DC (1980) Changes in endolymphatic potential and crossed olivocochlear bundle stimulation alter cochlear mechanics. Science 210:71-72

Murugasu E, Russell IJ (1996) The effect of efferent stimulation on basilar membrane displacement in the basal turn of the guinea pig cochlea. J Neurosci 16:325-332

OATMAn LC (1971) Role of visual attention on auditory evoked potentials in unanesthetized cats. Exp Neurol 32:341-356

Özdamar Ö, Dallos P (1976) Input-output functions of cochlear whole-nerve action potentials: interpretation in terms of one population of neurons. J Acoust Soc Am 59:143-147

Robles L, Delano PH (2008) Efferent system. In: Dallos P, Oertel D (eds) The senses: a comprehensive reference. Academic Press, London, pp 413-445

Siegel JH, KIm DO (1982) Efferent neural control of cochlear mechanics? Olivocochlear bundle stimulation affects cochlear biomechanical nonlinearity. Hear Res 6:171-182

SoHmer H (1965) The effect of contralateral olivo-cochlear bundle stimulation on the cochlear potentials evoked by acoustic stimuli of various frequencies and intensities. Acta Otolaryngol 60:5970

Sridhar TS, Liberman MC, Brown MC, Sewell WF (1995) A novel cholinergic "slow effect" of efferent stimulation on cochlear potentials in the guinea pig. J Neurosci 15:3667-3678

Teas DC, Konishi T, Nielsen DW (1972) Electrophysiological studies on the spatial distribution of the crossed olivocochlear bundle along the guinea pig cochlea. J Acoust Soc Am 51:1256-1264

WARR WB, GUINAN JJ JR (1979) Efferent innervation of the organ of corti: Two separate systems. Brain Res 173:152-155

Wiederhold ML (1970) Variations in the effects of electric stimulation of the crossed olivocochlear bundle on cat single auditory-nerve-fiber responses to tone bursts. J Acoust Soc Am 48:966-977

Wiederhold ML, Peake WT (1966) Efferent inhibition of auditorynerve responses: Dependence on acoustic-stimulus parameters. J Acoust Soc Am 40:1427-1430

Zheng XY, McFadden SL, Henderson D, Ding DL, Burkard R (2000) Cochlear microphonics and otoacoustic emissions in chronically de-efferented chinchilla. Hear Res 143:14-22 